\title{
LOS TRATADOS INTERNACIONALES Y EL SISTEMA DE FUENTES DE DERECHO EN EL PERU
}

\author{
Guillermo Fernández-Mald onado C.*
}

\section{Introducción}

Cumplida la primera década de vida de la Constitución peruana de 1979, resulta propicio profundizar nuestra reflexión sobre su contenido y vigencia. Con este propósito, dentro de la materia específica de los tratados internacionales, intentaremos señalar las notas que, a nuestro juicio, son las más relevantes de la normativa constitucional en relación con el sistema de fuentes de derecho, poniendo al descubierto los aspectos más problemáticos del tema.

Debo advertir que este trabajo se ha realizado desde una óptica poco usual, que puede plantear muchos más problemas que soluciones, mostrando contradicciones y oscuridades que están en el propio texto constitucional, aunque pocos parecen haber reparado en ellas.

La regulación constitucional de los tratados es un tema apasionante pero poco estudiado en nuestro medio, pese a que nuestra Carta introduce preceptos novedosos y avanzados en esta materia. Por regla general, nuestros constitucionalistas no han prestado mayor atención a la problemática de la acción exterior del Estado y, entre sus múltiples aspectos, a los tratados internacionales. Esto ha sucedido, probablemente, en el entendido que se trata de una labor que corresponde realizar a los internacionalistas; éstos, a su

(*) El presente trabajo es una reelaboración del que realizara a propósito del XX Curso Exterior de la Academia de Derecho Internacional de La Haya, celebrado en Bogotá, en junio de 1989. 
vez, han preferido analizar los tratados en el marco doctrinal y siguiendo lo dispuesto por la Convención de Viena de 1969 sobre el derecho de los tratados, donde la referencia constitucional es accesoria. Existe, pues, un paralelismo a todas luces perjudicial.

Muchos constitucionalistas entienden que los avances del Derecho Internacional son, en el fondo, derrotas que se infringen a la soberanía del Estado, por lo que tradicionalmente se han resistido a ellos. Este ha sido el trasfondo de las disputas doctrinales entre las tesis monistas y dualistas o, más modernamente, respecto de la constitucionalidad de la normativa comunitaria europea. Por su parte, los internacionalistas hallan en la soberanía estatal el principal obstáculo para el desarrollo del Derecho Internacional.

Esta realidad, antes que fomentar el análisis parcial desde cada especialidad, evidencia la necesidad de conjugar esfuerzos multidisciplinarios para comprender en su real dimensión estos temas, para lo cual es preciso reforzar la formación constitucional de los internacionalistas y viceversa.

Existen aspectos que tradicionalmente han sido retenidos en la esfera internacional pero que, al interactuar o conjugarse con otros, resulta imprescindible su estudio desde otra perspectiva, como la constitucional y de la ciencia política. Temas como la democratización y la acción exterior del Estado, los órganos de representación, gestión y control de la acción exterior, la constitucionalización de los principios internacionales, la Constitución como límite a la acción exterior, el papel del Ejecutivo y el Congreso en las diversas fases de la celebración y denuncia de los tratados o el control de la constitucionalidad de éstos, son sólo algunos ejemplos.

\section{La regulación general de los tratados en la Constitución peruana}

La Constitución peruana de 1979 contiene un capítulo exclusivamente reservado a la regulación de los tratados internacionales, normando de forma bastante más amplia y detallada que las cartas precedentes, los diversos aspectos relacionados y adoptando, ex- 
presa o tácitamente, muchos de los principios que hoy rigen en el derecho internacional (1).

La especial atención prestada por los constituyentes a estos instrumentos internacionales, revela la importancia adquirida en la actualidad y constituye un significativo avance en torno a diversos problemas derivados de la oscuridad o simple omisión que caracterizaba su regulación constitucional previa; pero, además, se caracteriza por haber otorgado a los tratados de "una importancia y' jerarquización importante dentro de nuestro sistema juridico" (2).

El contenido de las normas constitucionales pertinentes, plantea una sugerente forma de regulación, estableciendo una serie de distinciones que están dirigidas a valorar en diverso grado a los tratados guiándose, en algunos casos, por la materia a la que estén referidos. En otras palabras, la materia regulada informará su relación jerárquico-normativa con otros tratados y las normas internas. Estas son las líneas maestras que organizan y orientan la regulación constitucional de los tratados que, a continuación, pasamos a examinar.

\section{Concepto y alcance del término "tratado" en la Constitución}

Existe relativo consenso en entender por tratado al acuerdo de voluntades realizado entre sujetos de derecho internacional, dirigido a producir efectos jurídicos y regulado por el derecho internacional (3).

(1) Una visión sistemática de los textos constitucionales peruanos puede hallarse en UGARTE DEL PINO, Vicente. Historia de las Constituciones del Peru. Edit. Campodónico, Lima, 1971 y en PAREJA PAZ-SOLDAN, José. Derecho Constitucional Peruano. Studium, Lima, 1973.

(2) RUBIO CORREA, M. y BERNALES, E. Peri: Constitución y Sociedad Politica. Mesa Redonda Edit., Lima, 1981, p. 313.

(3) Véase, entre otros, a REUTER, P. Instroducción Droit des Traités, París, 1972, pp. 39 y ss.; JIMENEZ DE ARECHAGA, E. Curso de Derecho Internacional Público. T. I, Montevideo, 1959, p. 98; DIEZ DE VELAZCO, M. Instituciones de Derecho Internacional Público. T. II, Tecnos, Madrid, 1984, p. 96; SORENSEN, M. Manual de Derecho Internacional Público. México, 1973, p. 155, etc. 
La definición de tratado que aparece en el artículo 2, párrafo 1 literal a) del Convenio de Viena de 1969 sobre el derecho de los tratados - dirigida a establecer el ámbito de su aplicación-, contiene dos limitaciones al concepto antes expuesto:

\section{"Articulo 1.- Para los efectos de la presente Conven- ción:}

a) se entiende por tratado un acuerdo internacional celebrado por escrito entre Estados y regido por el derecho internacional, ya conste en un instrumento único o en dos o más instrumentos conexos y cualquiera que sea su denominación particular "”.

La primera restricción consiste en que sólo considera como tratados a los acuerdos que adoptan la forma escrita. La segunda, cuyo contenido limitativo es mucho más importante, se plasma en el hecho de que reconoce como sujetos de derecho internacional capaces de celebrar tratados sólo a los Estados, excluyendo así a las organizaciones internacionales (4).

No obstante, debemos notar que las restricciones que incorpora el concepto de tratado de la Convención de Viene de 1969 operan, únicamente, a los efectos de la aplicación de sus disposiciones y no restan validez jurídica a los tratados que escapan a su esfera de aplicación directa.

En nuestro pais, a pesar que la práctica internacional peruana, en los hechos, está orientada por las normas de la Convención de Viena, las obligaciones jurídicas en cuanto a la observancia obligatoria de tales reglas no son exigibles, pues el Perú no ha ratificado aún dicho instrumento.

(4) Inicialmente la Comisión de Derecho Internacional no realizó esta exclusión, su posterior incorporación en el texto definitivo de la Convención, asi como el retiro de la propuesta de los Estados Unidos, obedecen a las complicaciones y retraso que supondria regular los peculiares tratados con organizaciones internacionales, y no a una tácita adopción de la tesis soviética respecto de estas organizaciones. Véase: A nuario de la Comision de Derecho Internacional, 1966, Vol. II, p. 206 y ss. 
Existe relativo consenso en la doctrina jurídica nacional en el sentido que la Constitución atribuye el mismo significado y alcance a los tratados y a los convenios. Para sostenerlo se puede citar el art. 210.14, donde dice que el Presidente de la República tiene entre sus facultades la de "... .celebrar y ratificar tratados y convenios de conformidad con la Constitución".

Sin embargo, esta primera afirmación debe ser contrastada con un análisis más detenido del significado de convenio y tratado que se desprende del articulado del Capítulo $\mathrm{V}$ de la Constitución, "De los Tratados". Como detallamos más adelante, el término "convenio" se utiliza para designar los tratados que celebra y ratifica el Presidente en materias de su exclusiva competencia y sin la previa intervención del Parlamento. Por otro lado, el artículo 101 de la constitución, al referirse a los tratados, alude únicamente a los celebrados por el Perú "con otros Estados". La presencia de los organismos internacionales como sujetos de derecho internacional, según nuestra Constitución, se reduce al ámbito de los convenios regulados por el artículo 104. Estas consideraciones apoyan la tesis de que, en los tratados que requieren preceptivamente de la aprobación del Parlamento, es decir, aquellos que modifican o necesitan desarrollos legislativos, no podrían ser parte las organizaciones internacionales.

Otro aspecto de especial importancia de la definición de tratado inicialmente transcrita, es el relativo a la denominación utilizada. El concepto que adopta la Convención de Viena de 1969 señala con claridad que las características jurídicas del tratado se mantienen "cualquiera sea su denominación particular", codificando de esta forma la postura generalizada que sostiene que "la diversidad terminológica es jurídicamente irrelevante" (5).

Es cierto que históricamente los tratados han adoptado múltiples denominaciones, no obstante (6), "la diferencia formal entre

(5) VERDROSS, A. Derecho Internacional Público, Madrid, 1978, p. 129.

(6) PORTOCARRERO OLAVE, F. Derecho Internacional Publico. Ediciones Peruanas, Lima, 1966. 
acuerdo, acta, protocolo, convenio, convención o tratado no tiene ninguna importancia desde el punto de vista del valor internacional de las obligaciones contraídas".

Atendiendo a todo ello y desde la perspectiva del derecho internacional, las denominaciones que sean adoptadas por las partes al celebrar un acuerdo con las características anotadas, no afectan la naturaleza ni los efectos jurídicos del mismo. Esta situación puede variar cuando se ingresa dentro de la esfera del derecho interno, pues las normas constitucionales pueden realizar una distinción entre tratado y convenio que no es sólo nominativa, sino que tiene relevancia jurídica para el ordenamien to interno, correspondiéndoles materias, sujetos y procedimientos diversos, temas que reseñaremos a continuación.

\section{Celebración y entrada en vigor}

El artículo 211 de la contitución, en donde se establecen las atribuciones y obligaciones del Presidente de la República, dispone en su numeral 14:

"Articulo 211.- Son atribuciones y obligaciones del Presidente de la República:

14.- Dirigir la política exterior y las relaciones internacionales, y celebrar y ratificar tratados y convenios de conformidad con la Constitución".

La norma utiliza de forma indistinta el término convenio o tratado, pese al tratamiento diferenciado que se sugiere en el Capítulo $\mathrm{V}$ de la Constitución. La presencia de ambos términos puede explicarse por el hecho de que el Presidente de la República participa en los distintos procedimientos establecidos por la Carta, para la celebración y entrada en vigor de ambos instrumentos.

No obstante, este razonamiento podría resultar insuficiente para otras normas constitucionales que utilizan indistintamente los términos convenio, pacto o protocolo, cuando aluden a la categoría de "tratado" que hemos esbozado, como es el caso del art. 99 
y las disposiciones generales décimo sexta y décimo sétima. No obstante, estas normas aluden a acuerd os internacionales particulares que llevan tal denominación: Convención del Mar, Pacto Internacional de Derechos Civiles y Políticos, Protocolo Facultativo del Pacto Internacional de Derechos Civiles y Políticos de las Naciones Unidas, Convención Americana sobre Derechos Humanos de San José de Costa Rica, Convenio 151 de la OIT, etc., y no al "tratado" o "convenio" como categorías generales en la Constitución.

\subsection{El caso de los tratados}

Normalmente cuando existe acuerdo entre las partes sobre los términos de un futuro tratado, fruto de la previa negociación, los representantes de los Estados "adoptan" el texto del mismo, para luego concluir en su autenticación.

En el Perú, la Cancillería registra y archiva el texto del tratado a autenticado, dejando que el impulso hacia su perfeccionamiento proceda del sector ministerial respectivo, procedimiento que ha provocado más de una crítica por contribuir a la ya excesiva morosidad en estos trámites (7).

Sin duda, la etapa de mayor complejidad del proceso de celebración de los tratados es aquella en la que los Estados manifiestan su consentimiento, es decir, expresan su voluntad de obligarse en los términos del mismo. El carácter esencialmente interno de esta etapa, hace que las disposiciones de derecho internacional sean bastante genericas, dejando al derecho nacional su regulación específica.

Siguiendo la práctica extendida en la mayor parte de los países, la Constitución de 1979 establece dos instancias, dirigida la primera a la aprobación por parte del Parlamento, requisito necesario para que el Presidente de la República, en una segunda instan-

(7) DE LA LAMA EGGERSTEDT, M. La Constitución politica de 1979 y' los Tratados, en: La Constitución peruana de 1979 y sus problemas de aplicación. Eguiguren, F. compilador. Edit. Cuzco, Lima, 1987. 
cia, proceda a la ratificación respectiva. El fundamento constitucional se halla en el artículo 102 de la Carta: "Todo tratado internucional debe ser aprobado por el Congreso, antes de su ratificación por el Presidente de la República", disposición cuyo contenido es reiterado en los artículos 186.3 y 211.14 , relativos a las a tribuciones del Congreso y a las atribuciones y obligaciones del Presidente de la República, respectivamente.

La intervención del Parlamento se efectúa en ejercicio de su facultad de control, expresiva de la vigencia de la división de poderes (8). El objeto de este medio de control es que el tratado sea aprobado "de conformidad con la Constitución" (art. 186 inciso tercero), compatibilidad que también se verifica respecto de los compromisos internacionales contraídos previamente por el país y de la legislación interna. Esta aprobación es necesaria, por otro lado, pues el Parlamento pierde cierto margen de competencia legislativa, en la medida en que el tratado prevalecerá sobre las leyes aprobadas y por aprobarse por el Congreso.

La política exterior se ha visto democratizada en el presente siglo. Locke, Rousseau, Tocqueville, reconocían que la acción exterior precisaba de mayor libertad y flexibilidad para el Ejecutivo, por lo que el Parlamento, debido al número de representantes, su inestabilidad y heterogeneidad, además de tradicional lentitud, debia abstenerse de participar salvo en aspectos puntuales, como la conclusión de tratados que afectasen la vida, la hacienda o las leyes, así como los referidos a la declaración de guerra.

Esta postura ha prevalecido en el constitucionalismo decimonónico y fue posteriormente reelaborada para sostener doctrinariamente regímenes autoritarios, como ha sido el caso de la tesis del "poder exterior" (auswartige Gewalt) de E. Wolgast en el Tercer Reich o como fluye de la Ley Constitutiva de las Cortes de 1942 y la Ley Orgánica de Estado de 1967, de la dictadura de Franco en España.

(8) Véase MELo VEGA, J. Control Parlamentario en: La Constitución peruana de 1979 y sus problemas. op. cit., pp. 497 y ss. 
Nuestra Constitución ha desechado la tesis autoritaria, optando por la democratización de la política exterior que, en palabras de REMIRO BROTONS (9) "implica hacer efectivo el principio de la soberanía popular en el ámbito de la política exterior del Estado, hasta el limite en que su naturaleza lo permita".

La aprobación por el Congreso se plasma en Resoluciones Legislativas, numeradas correlativamente a las leyes. Sobre su naturaleza jurídica aún no hay acuerdo en la doctrina nacional, pues algunos le asignan el rango de ley (10). mientras que otro sector sostiene que se trata de actos administrativos del Congreso (11). Un elemento a tomar en consideración en el momento de tomar partido por una de estas posturas, lo constituye el hecho de que la aprobación congresal es la que brinda legitimidad a los tratados para que puedan imponerse a las leyes en caso de colisión normativa, tesis que plantea problemas si se sostiene que se trata de simples actos administrativos.

Debemos subrayar también que las referidas Resoluciones Legislativas se limitan a mencionar la aprobación de determinado tratado, pero el texto del instrumento internacional no es publicado. sino que pasa a ser archivado en la Cancillería. Esta omisión es importante, en la medida que si estos tratados, según el art. 97 de la Carta, forman parte del derecho nacional, su publicidad es esencial para su existencia, como expresamente establece el art. 87 in fine de la Constitución. Al respecto, tampoco parece aconsejable publicar el texto del tratado conjuntamente con la Resolución Legislativa, pues podría crróneamente creerse que es desde su publicación que se calcula la vigencia del tratado. cuando en realidad es preciso que se verifiquen otros actos, como la ratificación presidencial.

La ratificación que realiza el Presidente de la República, de-

(9) RI:MIRO BROTONS, A. L, a accion exterior del Estado. Tecnos, Madrid, 1984, p. 16.

(10) RUBIO/BERNALES. Peri: Constitución. . . op. cit., p. 313.

(11) DE. LA LAMA E., M. La Constitución. . op. cit. pp. 477 y ss. 
biera ser el acto mediante el cual las normas de derecho internacional convencional contenidas en el tratado, ingresan a formar parte del ordenamiento jurídico interno. No obstante, la ratificación no es suficiente para que el Estado se vea obligado internacionalmente, es necesario todavía que manifieste al ex terior su consentimiento. En efecto, es preciso que la ratificación o, en su caso, la adhesión, sea comunicada a las demás partes del tratado. Incluso es posible que la comunicación mediante el intercambio o depósito del instrumento de ratificación, no baste para la entrada en vigor del tratado y, en consecuencia, para que las obligaciones que contiene el instrumento sean exigibles entre las partes.

Finalmente, como se sabe, un tratado entra en vigor en la fecha que en él se disponga o acuerden las partes, tal como aparece dispuesto en el artículo 24 de la Convención de Viena de 1969 , aunque en los tratados multilaterales, es frecuente que la entrada en vigencia se supedite al depósito de un determinado número de instrumentos de ratificación.

En tal virtud, mientras que el acuerdo de voluntades que supone el tratado no se perfeccione $\mathrm{y}$ tenga vigencia efectiva, no existe tratado en propiedad y las normas constitucionales relativas a los "tratados" no pueden ser aplicadas.

3.2. Los convenios sobre materias de competencia exclusiva del Presidente de la República

Como ha sido subrayado, para el derecho internacional, la denominación particular que pueda asignarse a un acuerdo de voluntades entre sujetos de su esfera y sometidos a su regulación, no reviste relevancia jurídica alguna. Siempre que se compruebe que el objeto del acuerdo es la producción de efectos jurídicos, regulados por el derecho internacional, se está en presencia de un tratado internacional.

Por esta razón, la distinción que se realiza en el derecho nortamericano entre "treaties" y "executive agreements", análogamente a lo que se opera en el Perú respecto a los "tratados" y 
"convenios", debiera revestir importancia sólo a efectos del derecho interno.

Esta distinción, que la doctrina conoce como tratados formales y acuerdos en forma simplificada, valora en los segund os su ágil expedición y la ausencia de la intervención previa del Congreso, tal como dispone el artículo 104 de la constitución:

"Artículo 104. - El Presidente de la República puede, sobre materias de su exclusiva competencia, celebrar o ratificar convenios internacionales con Estados extranjeros $u$ organizaciones in ternacionales o adherir a ellos sin el requisito previo de la aprobación del Congreso. En todo caso debe dar cuenta inmediata a éste".

El precepto constitucional peruano ha optado por el sistema de "lista negativa", es decir, los acuerdos internacionales que no son materia exclusiva del Presidente de la República precisan la aprobación previa del Parlamento; es una competencia de carácter residual que tiene algunos inconvenientes y complicaciones.

Parece mejor el sistema de "lista positiva", plasmado, por ejemplo, en el artículo 94.1 de la carta constitucional española, que establece los casos en que se requiere la intervención del Parlamento. La norma española cuida de señalar los tratados que, necesariamente, deben contar con la previa autorización de las Cortes Generales para la prestación del consentimiento del Estado: los de carácter político, militar, que afecten la integridad territorial o los derechos fundamentales, que impliquen obligaciones financieras para la Hacienda Pública y, finalmente, los que supongan modif icación o derogación de alguna ley o exijan medidas legislativas para su ejecución. En esta línea se hallan el art. 53 de la Constitución francesa, el art. 80 de la Constitución italiana, 59.2 de la Ley Fundamental de Bonn de la República Federal de Alemania, etc.

Los acuerdos en forma simplificada, caracterizados por tener un procedimien to más ágil y menos solemne, se celebran mediante un canje de notas, memoranda de entendimiento, minuta aprobada o por convenios con una redacción de menor solemnidad que la que se dispensa a los tratados clásicos. En el caso peruano, la ra- 
tificación por parte del Presidente de la República se materializa en una Resolución Suprema.

Los problemas que plantea este procedimiento se enmarcan estrictamente en la esfera del derecho interno, ya que los efectos hacia el exterior no sufren modificación alguna. Ello obedece a que las normas de la Convención de Viena son bastante amplias en esta materia y se remiten, básicamente, a las condiciones que contenga el propio tratado, al acuerdo e intención de las partes y al derecho interno de cada Estado (12).

Así, en el ámbito interno, la dificultad principal se centra en establecer los criterios para diferenciar los tratados que requieren de la aprobación previa del Congreso, de los que pueden ser celebrados y ratificados por el Presidente de la República sin este requisito previo. La importancia de esta determinación estriba en que en virtud de tal operación, se podrá conocer el valor normativo que le corresponde a dicho texto legal en el sistema de fuentes de derecho interno. Esto, en el entendido que los convenios se hallan por debajo de las leyes y ningún precepto constitucional reservado a los tratados le resulta aplicable.

El enunciado del artículo 104 de la Constitución resulta excesivamente genérico cuando intenta delimitar un ámbito competencial para los convenios. pues no llega a definir el alcance de "las materias de exclusiva competencia del Presidente de la República". Al parecer, el constituyente juzgó que con esta fórmula no podría generarse un conflicto normativo, pues tanto el Presidente de la República como el Parlamento actuarian dentro de materias reservadas.

Un primer aspecto a destacar es que, en rigor, el Presidente de la República carece de competencias exclusivas, es decir, competencias que pueda ejercer por sí $v$ ante sí. En efecto, nuestra Constitución establece la irresponsabilidad política del Jefe de

(12) Esta materia se halla regulada básicamente por los artículos 12 al 14 de la Convención de Viena de 1969. 
Estado y, en tal virtud, el art. 213 de la Carta sanciona con nulidad los actos del Presidente de la República que carezcan de la correspondiente refrendación ministerial.

Dejando de lado lo anterior, las materias en las que puede existir competencia exclusiva del Presidente, que el texto constitucional señala de forma dispersa y poco clara, no se hallan necesariamente fuera del campo de acción de la labor legislativa. Veamos por ejemplo la potestad reglamentaria, característica del Poder Ejecutivo y recogida en el tex to constitucional:

"Articulo 211. - Son atribuciones y obligaciones del Presidente de la República:

11.- Ejercer la potestad de reglamentar las leyes sin transgredirlas ni desnaturalizarlas; $y$, dentro de tales límites, dictar decretos y resoluciones".

Admitiendo que ésta podría ser una materia de exclusiva competencia del Presidente de la República, ello no obsta para que el Parlamento legisle regulando en la forma y en el fondo diversos aspectos relativos a la potestad reglamentaria. En otras palabras, cl ejercicio de esta potestad es exclusivo del Presidente, pero su regulación legal corresponde al Parlamento.

Veamos otro ejemplo extraído del tex to constitucional:

"Artículo 211.- Son atribuciones. . .

22.- Regular las tarifas arancelarias".

Según esta norma, la regulación arancelaria es exclusiva del Presidente de la República y, de existir un acuerdo in ternacional sobre esta materia, éste debiera entrar dentro de la categoría de convenios que la constitución reconoce, con los efectos jerárquiconormativos consecuentes. No obstante, la gran mayoría de tratados sobre integración y muchos de cooperación incluyen normas relativas al desarme arancelario temporal o paulatino.

La confusión crece cuando notamos que el artículo 104 es 
potestativo: "El Presidente de la República puede. ..". En tal sentido, siendo una atribución facultativa, el Presidente puede no clegir la vía del 104, optando por el procedimiento que corresponde a los tratados. Esto es perfectamente posible y constitucional, de modo que el resultado será un tratado sobre materias de exclusiva competencia presidencial. Esta hipótesis demuestra la insuficiencia del principal elemento diferenciador que propone el texto constitucional, a saber, las materias de exclusiva competencia del Presidente de la República.

Así, la "exclusiva competencia" presidencial sólo informa sobre las materias en las que el Presidente de la República puede utilizar el ágil procedimiento que consigna el artículo 104, diverso al que constitucionalmente corresponde para los tratados $\mathrm{y}$, por ende, no todo acuerdo que regule materias que ingresen dentro de tal competencia presidencial deben, automáticamente, ser clasificados como convenios.

Dado que el propio tex to constitucional no nos puede dar luces suficientes para dar solución a este problema, se ha intentado aproximarse doctrinalmente a él (13), señalando que la fórmula constitucional "implica los acuerdos meramente ejecutivos, administrativos y reglamentarios". Postura análoga fluye de la clíusula general contenida en el artículo 94.1 in fine de la Constitución ispañola, al definirlos como aquellos acuerdos internacionales que no modifiquen la legislación nacional y que no exijan leyes complementarias para su ejecución.

De esta forma, el método más simple para determinar si se trata de un tratado o un convenio, no ingresa a analizar la materia, sino se refiere únicamente a la norma interna que lo contiene. $\mathrm{Si}$ se trata de una Resolución Legislativa se está ante un tratado, si es una Resolución Suprema se trata de un convenio.

No obstante, este método es básicamente formal y tiene sentido si efectivamente se respeta lo dispuesto por el artículo 104,

(13) DE LA LAMA EGGERSTEDT, M. La Constitución. . . op. cit., p. 484. 
es decir, optar por la vía simplificada sólo cuando se trata de materias de exclusiva competencia del Presidente. Por ello, considerando que es el propio Poder Ejecutivo el que decide si se trata de un acuerdo sobre estas materias y, en tal virtud, aprueba o ratifica el convenio sin la aprobación previa del Parlamento, resulta necesario que se ejerza un control sobre tal calificación y actuación, así como sobre su legalidad y constitucionalidad.

Es cierto que el Parlamento, cuando estudia el texto de un tratado para su aprobación, debe ejercer un control sobre su constitucionalidad, pero éste sólo puede llevarse a cabo si el Ejecutivo decide remitirle el texto para su aprobación. El control parlamentario a posteriori aparece consignado en el artículo 104 in fine de la Constitución, que está referido únicamente a los convenios celebrados y ratificados por el Presidente, de los que en todo caso debe darse cuenta al Congreso. Sin embargo, se trata de un control escasamente realizado en la práctica y que parece dirigirse a un hecho consumado.

A diferencia de lo que ocurre en otros Estados, en el Perú no existen de forma expresa controles preventivos ni reparadores sobre la constitucionalidad de los acuerdos internacionales, sea sobre el fondo o la forma. El art. 298 de la Constitución, al referirse a las competencias del Tribunal de Garantías Constitucionales,' señala que puede declarar, a petición de parte, la inconstitucionalidad parcial o total de las leyes, decretos legisla tivos, normas regionales de carácter general y ordenanzas municipales que contravienen la Constitución por la forma o por el fondo, enumeración taxativa que no alude a los tratados o los convenios internacionales, así como tampoco a las Resoluciones Legisla tivas.

En España, por ejemplo, el Tribunal Constitucional, conforme al art. 95.2 de la Constitución, está encargado de declarar la inconstitucionalidad material de un tratado internacional, decisión que es obligatoria y previa a su aprobación y ratificación. En los casos en que el tratado ya ha sido aprobado por el Parlamento, el art. 27.2 de la Ley Orgánica del Tribunal Constitucional incorpora a los tratados entre las normas susceptibles de ser declaradas inconstitucionales, pese a que el texto constitucional no lo señala 
de forma expresa. Se debería estudiar la posibilidad de subsanar la omisión que también se presenta en nuestra Carta, mediante su expresa incorporación en la Ley Orgánica del Tribunal de Garantías Constitucionales.

\section{Valor normatiro de los tratados internacionales}

Las normas constitucionales han establecido ciertas precisiones en torno al valor de los tratados internacionales, que están dirigidas a dar pautas en los casos que puedan existir conflictos con leyes internas, con otros tratados y con la propia Constitución.

E1 contenido de estos preceptos configuran un sistema de relaciones normativas, donde confluyen consideraciones de orden jerárquico y competencial. La regla general es la de otorgar una situación de privilegio a los tratados internacionales dentro del sistema de fuentes de derecho interno. El sustento de estas normas constitucionales es el reconocimiento de la naturaleza propia del derecho internacional convencional, el cual posee un carácter "ius supra partes". Como señala Santi Romano (14), "el acuerdo normativo produce una voluntad que no es la de los Estados en particular, sino una voluntad distinta, única, en la que aquélla se funda y que es superior a ellas".

De esta forma, se garantiza de forma muy amplia la aplicación preferente de las normas de derecho convencional internacional en la esfera interna, reconocimiento que se traslada del campo de la simple interpretación al de su consagración explícita en la lex superior del derecho interno, a saber, la Constitución.

Para mostrar con mayor claridad el alcance de las normas constitucionales que regulan esta materia, hemos considerado adecuado reseñar las soluciones que resultan de su aplicación sobre posibles conflictos normativos.

(14) SANTI ROMANO, $k l$ ordenamiento juridico. Madrid, 1963, p. 145. 


\section{El conflicto entre tratado y ley interna}

Según establece el tex to constitucional:

"Artículo 101.- Los tratados internacionales celebrados por el Perú con otros Estados, forman parte del derecho nacional. En caso de conflicto entre el tratado y la ley, prevalece el primero".

La norma constitucional reconoce el tratado como parte del ordenamiento interno para, a continuación, indicar su ubicación jerárquica dentro del sistema de fuentes de derecho. Siguiendo este planteamiento, la doctrina nacional entiende, de forma unánime, que los tratados están situados dentro de la jerarquía que corresponde a las leyes (15).

Sin embargo, cuando en este supuesto se presente una colisión normativa, en principio entre normas de igual jerarquía, la solución no resulta de la aplicación del principio "lex posterior derogat priori". En ef ecto, los constituyentes, atendiendo a que los tratados suponen una obligación del Estado con el exterior, les otorgaron prevalencia sobre las demás leyes internas que se le opongan.

De esta forma, las leyes internas que contengan normas que se hallen en contradicción con las disposiciones de un tratado dejarán de aplicarse en favor de éste $y$, por otro lado, las leyes que se aprueben con posterioridad a la entrada en vigencia de un tratado, tampoco podrán tener efectos jurídicos que puedan impedir la aplicación del instrumento internacional.

Nótese que en este caso la prevalencia no tiene explicación suficiente en razón de la jerarquía, sino que es necesario atender a criterios de competencia. No se trata, sin embargo, que median-

(15) Véase la postura de RUBIO/BERNALES y DE LA LAMA, en los tex tos citados y CHIRINOS SOTO, E. La Constitución al alcance de todos, $\mathrm{Li}$ ma, 1984, p. 114. 
te esta norma de aplicación general se reserva materias que ingresan dentro de la exclusiva competencia de los tratados. Las leyes internas no pierden total competencia en tales materias, el Parlamento puede legislar, pero si existe colisión será de aplicación el principio constitucional de prevalencia de los tratados.

En realidad se trata de regulaciones que apuntan a establecer los criterios que deben utilizarse para elegir la norma aplicable. Al respecto resulta necesario efectuar dos precisiones en torno al valor normativo de los tratados como regla general de aplicación. En primer término, se asigna a los tratados.un valor jerárquico equivalente al de las leyes, llegando a atribuírseles los efectos que el derecho interno reserva a las leyes sobre las normas inferiores jerárquicamente, incluso el efecto derogatorio (16). Sobre este último punto descubrimos varios problemas e interrogantes, por ejemplo, en el caso de verificarse la derogación, ¿qué normas inferiores a la ley serían aplicables en los casos en que se trate de sujetos que no son parte del tratado o no pueden invocarlo?, ¿en qué situación quedan dichas normas luego que el tratado finaliza por cumplirse su plazo de vigencia o por denuncia efectuada por el Presidente de la República?

En segundo lugar, atendiendo a criterios de competencia, se reconoce la prevalencia de las normas contenidas en tratados internacionales sobre aquellas normas dispuestas en leyes internas que se le opongan. En este caso, se trata de una relación horizontal y no vertical como la jerárquica, razón por la que sus efectos no son derogatorios sino de inaplicabilidad.

Es así que el tratado no deroga los preceptos de una ky que le sean opuestos, únicamente los deja inaplieables a efecto de garantizar el eumplimiento de las obligatcones del Estado derivadas de su condición de parte del tratado. Fsta obligación sólo existe para los supuestos en que debe aplicarse el tratado. de modo que la ley mantiene plena vigencia sobre la misma materia que regula el tratado en los demás casos. La ley resulta aplicable, en todo caso, en virtud de que no existe contradicción con ningún tratidlo.

(16) CHIRINOS SOTO. F. I a muera constitucion. . op. cit. p. 114 
Ha quedado establecido con meridiana claridad, que la regla general es la de otorgar a los tratados una jerarquía equivalente a la de las leyes internas, aunque con prevalencia en caso de colisión normativa.

Siguiendo esta regla general, resultaría aplicable el principio de que un tratado posterior, celebrado entre las mismas partes. deroga el anterior. No obstante, si bien este principio mantienc vigencia plena respecto de las obligaciones existentes en la esfera del derecho internacional, la Constitución peruana contiene normas que establecen distingos entre los tratados según la materia que regulan, cuya principal consecuencia será la no aplicación de este principio en el derecho interno.

Antes hemos visto que cuando existe colisión normativa entre un tratado y una ley, el dato fundamental no es la materia que es regulada, sino la norma que contiene el mandato. En otras palabras, si existen preceptos que regulan una misma materia de forma contradictoria y éstos son recogidos por una ley y un tratado, respectivamente, deberá aplicarse el que figura en el tratado. De este modo, todas las disposiciones de una ley interna, sin distinción de la materia que regulen, quedan inaplicadas si entran en colisión con alguna disposición de un tratado, donde también resulta indistinta la materia en sí.

Cuando asistimos a una colisión normativa entre dos tratados, el razonamiento anterior ya no resulta aplicable. En efecto, conforme a nuestra Constitución, un mismo tratado puede contener normas a las que corresponde asignar distin ta fuerza normativa, determinación que se realiza atendiendo a la materia. Por esta razón, a diferencia de la colisión normativa tratado-ley, los efectos de la aplicación de la norma constitucional pueden dirigirse a artículos específicos que regulan materias específicas, mientras que la colisión de los demás preceptos del mismo tratado deberán ser resueltos haciendo uso de las reglas aplicables al conflicto entre normas de igual jerarquía, tal como veremos a continuación. 


\subsection{Los tratados que contienen normas relativas a los Derechos Humanos}

En abril de 1978, e1 Perú ratificó los Pactos sobre Derechos Civiles y Políticos y sobre Derechos Económicos, Sociales y Culturales aprobados en el marco de las Naciones Unidas en 1966, donde se desarrollan los derechos reconocidos en la Declaración Universal de los Derechos Humanos, de 10 de diciembre de 1948, la misma que fue incorporada al ordenamiento interno peruano de 1959. El Perú también ha ratificado el Protocolo Facultativo al Pacto de los Derechos Civiles y Políticos, así como la Convención Americana sobre Derechos Humanos de San José de Costa Rica.

En adición a la prevalencia que el artículo 101 de nuestra Constitución otorga a los tratados internacionales, los constituyentes juzgaron oportuno disponer una regulación más especial aún, en los casos que existan disposiciones relativas a los derechos humanos en los tratados internacionales, tal como queda plasmado en el artículo 105 de la Carta:

"Artículo 105. - Los preceptos contenidos en tratados relativos a derechos humanos, tienen jerarquía constitucional. No pueden ser modificados sino por el procedimiento que rige para la reforma de la Constitución".

Antes de ingresar al estudio de los posibles efectos de esta norma, es preciso hacer notar que la interpretación correcta de su alcance, a nuestro juicio, es que la norma está referida a los preceptos relativos a derechos humanos que estén contenidos en cualquier tratado, sin distinción. En tal sentido, si un precepto no tiene efecto ni relación alguna con la materia específica, aunque figure en un tratado que en su título y en la mayoría de su articulado sí se refiera a los derechos humanos, no puede considerarse que está dentro del campo de aplicación de la norma. Análogamente, si por ejemplo, un tratado sobre desarme o cualquier otra materia genérica contiene alguna disposición relativa a los derechos humanos, ésta se halla dentro del supuesto del artículo 105. Las demás normas de este tratado tendrán el valor jurídico que resulte de 
uplicar la regla general contenida en el artículo 101 de la Carta. Sostener lo contrario implicaría la desprotección de muchos preceptos que regulan la materia de derechos humanos, desnaturalizando el objetivo perseguido por la norma constitucional.

Los criterios que se utilizan para subrayar la importancia jurídico normativa de este tipo de tratados son, fuera de toda duda, de orden competencial. Se ha establecido esta regulación preferente "ratione materiae", considerando que lo que se pretende es privilegiar de forma especialísima determinadas normas de los tratados, en virtud de que regulan aspectos relativos a los derechos humanos. Por otro lado, esta interpretación resulta plenamente coherente con el énfasis que el resto del articulado constitucional coloca al regular estos derechos (17).

Si bien ésta es la vía adecuada para determinar a qué normas le corresponde aplicar el artículo 105 de la Carta, los efectos jurídicos de preeminencia no son de índole competencial sino jerárquico. En efecto, hemos visto que la Constitución establece, como regla genérica, que los tratados adquieren la jerarquía normativa de leyes internas, del mismo modo que dispone su prevalencia sobre éstas últimas cuando le son opuestas. En el siguiente apartado, al analizar los tratados de integración, descubriremos que nuestra Constitución también intenta establecer cierta prelación en función de campos competenciales específicos: la in tegración.

Sin embargo, ninguna de las reglas precedentes coincide con la elegida por el constituyente para el caso de las normas relativas a los derechos humanos. En este último supuesto, la prevalencia se sustenta en el otorgamiento de una jerarquía superior a la legal, se trata, como señala expresamente la norma, de una "jerarquía constitucional".

Como consecuencia de lo anterior, los efectos jurídicos que

(17) Sobre este tema véanse los estudios de ALIAGA ABANTO, O. y QUIROGA LEON, A., ambos en: La Constitución peruana de 1979 y sus problemas. ., op. cit. pp. 19-93 y 97-165. 
se derivan de semejante situación jerárquica difieren de los anotados para los tratados anteriores, tanto en el caso de un conflicto normativo entrc un tratado y una ley interna, como en el que si suscite entre dos tratados.

En este sentido, los efectos jurídicos derivados de la aplicación de la regla general, para los casos de colisión normativa con jerarquía de ley, ya no serán observados. Ello obedece a que la norma relativa a los derechos humanos contenida en el tratado, se le reconoce la jerarquía constitucional en el derecho in terno y, por ende, sus efectos sobre las normas inferiores que le sean opuestas, serán semejantes a los que se verifican en el caso de colisión Constitución-ley.

Es por esta razón que no sólo se dejaría inaplicada la ley contraria, como en el caso del conflicto tratado-ley, sino que podría a tacarse la validez de la norma interna opuesta, con el fin de lograr su expulsión del ordenamiento jurídico, siguiendo los cauces que la propia Constitución prevé para estos casos (18).

Somos de la opinión de que en este caso, dada la especial naturaleza de la materia regulada y la postura constitucional purceptible a lo largo de su articulado, no puede existir discriminación entre los que son parte del tratado y los que no lo son, de modo que las obligaciones que se derivan de estos instrumentos internaionales podrían ser exigidas sin distinción de los sujetos. La norma del tratado se aplicará en todos los casos en detrimento de las normas internas inferiores.

Por otro lado, cuando se trate de un conflicto entre normas de dos tratados. se aplicará la que sea relativa a los derechos humanos. Arribamos a esta conclusión apoyándonos, nuevamente. en la jerarquía constitucional otorgada a estas normas, por lo que

(18) Sobre el tratamiento general de las vias de control sobre las normas contrarias a la Constitución véase: SOUZA CALLE, M. y DANOS ORDO$\tilde{\mathrm{N}} \mathrm{EZ}, \mathrm{J}$. I: control jurisdiccional de la constitucionalidad. ... en I.a Constitucion peruana de 1979 y' sus. .., op cit. 
prevalecerán en pie de igualdad a u na norma constitucional. Resulta pertinente señalar lo dispuesto en el artículo 87 de nuestra Carta: "La Constitución prevalece sobre toda otra norma legal".

Fluye de lo anterior que, cuando existe una colisión normativa entre preceptos contenidos en tratados, a los cuales les corresponde una jerarquía de ley y constitucional, respectivamente. la solución es la prevalencia de la norma de mayor jerarquía en el ordenamiento interno, vale decir, la relativa a los derechos humalnos (19).

Desde otra perspectiva, debemos subrayar que dentro del supuesto del artículo 105 no están las normas relativas a los derechos contenidas en un tratado y otorguen menos derechos que los (jlic reconoce la propia Constitución. En efecto, en tal supuesto cxistiría una colisión entre un tratado y la Constitución, siendo de aplicación ló preceptuado en el artículo 103 de la Carta, que exige que su aprobación siga el procedimiento que rige la reforma constitucional, antes de la ratificación presidencial. Sólo la interpretación anterior explica por qué el artículo 105, establece que estas normas adquieren automáticamente la jerarquía constitucional. para luego disponer, como requisito necesario para su modificición. el seguir el procedimiento que corresponde a la reforma constitucional.

No obstante, subsisten algunas zonas oscuras sobre la situación normativa de estos preceptos. En primer término, el constituyente ha cuidado de subrayar la vocación de permanencia de 'stas normas, al descartar tácitamente la posibilidad de derogación y estableciendo de forma negativa la vía para su modificación: "No pueden ser modificados sino por el procedimiento que rige

119) Resultaria interesante analizar que sucede con las posibles responsabilidades del Estado, originadas por el incumplimiento de las obligaciones adquiridas en un tratado que no es aplicado gracias a la prevalencia de los que regulan materias relativas a los derechos humanos, según nuestro artículo 105, en especial si consideramos que resulta contrario a lo dispuesto en el artículo 27 de la Convención de Viena de 1969 para estos casos. 
para la reforma de la Constitución". Ello puede ser interpretado en el sentido que la aprobación congresal, necesaria para la denuncia del tratado que regula el artículo 107, debe seguir el procedimiento descrito. Sin embargo, ¿qué sucede en el hipotético caso que el tratado llegue a su fin por expiración del plazo? ¿Acaso las normas constitucionales se mantienen en vigor mientras no sean -modificadas según el procedimiento que establece la norma constitucional, vale decir, la reforma constitucional? Dejamos planteados estos problemas.

\subsection{Los tratados de integración}

El mejor modo de ingresar al tema de la regulación constitucional de los tratados de integración es transcribiendo una de sus normas, la que nos brindará la perspectiva adecuada para el análisis e interpretación de las demás normas del texto:

"Artículo 100.- El Perú promueve la integración económica, política, social y cultural de los pueblos de América Latina, con miras a la formación de una comunidad latinoamericana de naciones".

Tomando como marco este precepto, el constituyente dispuso posteriormente:

"Artículo 106. - Los tratados de integración con Estados latinoamericanos prevalecen sobre los demás tratados multilaterales en tre las mismas partes".

Esta norma no surte efectos adicionales a la solución ya planteada en los casos de conflicto entre el tratado y la ley, es decir, la inaplicación de la ley en favor de la norma del tratado. Sin embargo, sin que se extienda una jerarquía constitucional a los tratados de integración, éstos prevalecen sobre otros tratados en caso de colisión, siempre que se cumpla con los supuestos del artículo 106. En este último caso, la prevalencia en favor de un tratado sobre otro operaría como consecuencia de un reparto competencial específico: la integración. 


\subsubsection{La materia de la integración}

Debemos partir del supuesto de que la prevalencia que la Constitución otorga a estos tratados, opera en virtud de la materia que regulan y el objetivo que persiguen. Esto es, interesa privilegiar las materias incorporadas dentro de un proceso de integración y que están reguladas por un tratado de esta naturaleza.

En este sentido, para aplicar la norma constitucional, es preciso determinar previamente si las disposiciones del tratado son materia de un proceso de integración. Aquí encontramos un primer escollo, pues en rigor no existen materias exclusivas de las que pueda afirmarse posean una naturaleza integrativa típica. Por esta razón, el primer paso a seguir para determinar las materias abarcadas por un proceso de integración, es la referencia que las normas del propio tratado puedan señalar, incluso el grado de integración deseado en cada materia.

Así por ejemplo, en el Tratado de Montevideo de 1960, mediante el cual se crea la Asociación Latinoamericana de Libre Comercio (ALALC), se entiende que son materia de integración aquellas que están ligadas a la conformación de una zona de libre comercio, abarcando ciertas materias de índole económico, en especial los aspectos arancelarios.

Por otro lado, el Acuerdo de Cartagena no sólo plantea una zona de libre comercio, sino que aspira a consolidar una unión económica, por lo que se pueden hallar normas que regulan, dentro de los Estados, las materias que están comprendidas en "un proceso de coordinación de sus planes de desarrollo en sectores específicos y de armonización de sus políticas económicas y sociales, con la mira de llegar a un régimen de planificación conjunta para el desarrollo integrado del área" (20).

Fluye de lo anterior que no existen materias que puedan

(20) Artículo 26 del Acuerdo de Cartagena. 
exhibir autónomamente una naturaleza comunitaria o integrativa. Así por ejemplo, existen tratados de integración que regulan materias comerciales, pero existen también otros tratados no integrativos que regulan estas mismas materias.

Se puede ir más lejos aún, pues los tratados pueden resultar insuficientes como fuente que determine las materias que, en determinado momento, se hallan dentro del marco integrativo. Ello se debe a la particular naturaleza de la integración como "proceso", el cual presupone etapas o grados de integración. De esta forma, puede que existan materias que están consignadas en el tratado, pero aún no han sido desarrolladas o no son parte del proceso, no existiendo ninguna obligación para el Estado hasta el momento en que efectivamente se verifique dicho desarrollo.

También puede suceder que la dinámica del proceso de integración llegue a tal grado, que las competencias se extiendan hacia materias que no se hallan contenidas de forma explícita en el tratado. Como subraya Dagtolou (21), "el ámbito de la accion" comunitaria se extiende sin cesar, aunque con grados de intensidad diferentes".

En síntesis, las materias susceptibles de ser comprendidas por la integración no están preestablecidas, sino que son determinadas interpretando las normas de los tratados de integración y en su momento deben ser establecidas entendiendo la integración como "estado de cosas" y no como "proceso".

Dado que no puede existir una diferenciación autónoma entre materias de integración y las que no lo son, para determinar si una materia es de naturaleza integrativa no nos podemos guiar por la materia en sí, sino que debemos atender al sentido de su regulación. Al respecto reiteramos que dos tratados pueden regular materias similares y sólo uno de ellos encajar dentro de lo que se entiende por integración.

(21) DAgTOLOU, P. Naturaleza juridica de la Commidad limenera, en: Treinta años de Derecho Comunitarto. Comision de las Comunidades Europeas, Luxemburgo, 1984, p. 43 
Por otro lado, la naturaleza integrativa de un tratado depen derá de la existencia o no de principios reguladores integrativos. Así, de existir principios de carácter integrativo o comunitario, es decir, enmarcados dentro de un proceso gradual de integración en diversos sectores, el tratado ingresa en la categoría de los tratados de integración. A nuestro juicio, éste debe ser el criterio idónco para determinar si se está ante un tratado de integración y, en con secuencia, resulta aplicable la prevalencia establecida por el artículo 106 de la Constitución.

\subsubsection{Las Partes de un tratado de integración}

Para la prevalencia de los tratados de integración sobre otris tratados es preciso, además de la determinación de la materia, que estos últimos tengan carácter multilateral y sean celebrados entre las mismas partes, tal como aparece en la norma constitucional. En consecuencia, todo parece indicar que debe entenderse que los tratados bilaterales quedan fuera del supuesto planteado por la norma. Así por ejemplo, en el supuesto que un tratado bilateral, celebrado entre el Perú y uno de los países del Pacto Andino, colisione con normas del Acuerdo de Cartagena, éste, a pesar de tener carácter integrativo, no prevalecerá sobre el tratado bilateral, pues se estaría fuera del campo de aplicación del artículo 106 de la Carta.

Más de uno podría ensayar una interpretación constitucional más amplia de la norma, atendiend o a la voluntad objetiva e inmanente allí expresada (voluntas legis), considerando que su finalidad es la protección y garantía de los avances del proceso de integración que puedan contener este tipo de tratados. En efecto, no existe duda que la prevalencia normativa otorgada está justificada por la materia regulada y los principios orientadores. ambos ligados al proceso de integración, del que, constitucionalmente, el Perú es promotor, según se desprende de la lectura del artículo 100. Aceptemos esta hipótesis de trabajo.

Se deriva de lo anterior, que si no se admite en el derecho interno la inaplicación de un tratado de integración en virtud de otro tratai. multilateral, celebracio entre las mismas partes, pierde sen. tido la garantía de prevalencia si se admite que sólo dos Estadon 
Parte sí puedan imponerse a un tratado de integración. La interpretación literal de la norma no deja otra salida que una reforma constitucional, dirigida a armonizar el objetivo perseguido por el artículo 106, con los medios que establece para lograrlo.

Otro vacío de la norma es el relativo a los tratados celebrados con Estados que no son parte de un tratado de integración. En efecto, el artículo 106 no considera el caso de un acuerdo internacional que, celebrado con un tercer país, afecte el contenido y las obligaciones derivadas de un tratado de integración.

Al parecer, el constituyente no se colocó en esta hipótesis o, siendo consciente de esta posible colisión, decidió finalmente consignar la fórmula del artículo 106, la misma que no extiende la prevalencia de los tratados de integración. Este análisis podría complicarse si ingresamos a la problemática que se plantea en el caso del Acuerdo de Cartagena, pero es una materia que excede el objeto planteado para este trabajo (22).

\subsubsection{Alcance del Articulo 106}

El espíritu de la norma, además de lo que indica el mencionado artículo 100, puede sustentarse en dos supuestos diferentes. En primer lugar, se puede entender que los intereses nacionales resultan siempre compatibles o complementarios con el interés comunitario o, en segundo término, admitiendo posibles conflictos de intereses, se debe preferir siempre el interés integrativo. En todo caso, esto supone que se debe asumir los principios comunitarios como criterio de orientación, existiendo la posibilidad de efectuar interpretaciones no restrictivas de la norma (23).

(22) Véase sobre esta materia FERNANDEZ-M ALDONADO CASTRO, Guillermo. Aspectos Juridico-constitucionales de la Integración Andina. Tesis Doctoral. Universidad de Alcalá de Henares, 1987, p. 543 y ss.

(23) Una obra de consulta, en extremo útil en materia de interpretación jurídica, es la de DIEZ PICAZO, Luis. Experiencias juridicas y teoria del Derecho. Ariel, Barcelona, 1973. 
En efecto, una interpretación restrictiva, basada en el principio "quod non est in codice non est in mundo", podría originar una aplicación normativa que tenga, precisamente, los efectos que se intentan evitar mediante el artículo 106.

Con anterioridad hemos subrayado las ventajas que tendría una interpretación amplia del artículo 106, en especial en lo que concierne a su aplicación en los supuestos de "tratados multilaterales celebrados entre las mismas partes". No obstante, ante el peligro de generar un quiebre constitucional a través de una interpretación que podría desnaturalizar la norma en cuanto al ámbito de su aplicación alli señalado, es aconsejable seguir el procedimiento de reforma de la constitución para remediar estos vacíos $\mathrm{y}$ deficiencias.

\section{El conflicto entre el Tratado y la Constitución}

El ordenamiento jurídico peruano se organiza de forma análoga a la mayoría de países. La relación existente entre las distintas clases de normas se fundamenta en criterios básicamente jerárquicos (24). Esta es la pauta a seguir para determinar la norma que prevalece en caso de colisión. Dentro de este sistema jerarquizado de fuentes de derecho, la Constitución es, siguiendo a Pérez Royo (25), "fuente de derecho y norma que regula las fuentes de derecho", colocándose en una posición de supremacía jerárquica que es ampliamente reconocida en doctrina (26).

Por otro lado, la primacía jerárquica que corresponde a la constitución como "lex superior", se halla expresamente establecida en el propio tex to constitucional:

(24) Un estudio clásico sobre estos aspectos en el Perú fue realizado por FURNISH, Dale. La jerarquia del ordenamiento juridico peruano, en: Derecho No. 30, Pontificia Universidad Católica, Lima, 1972, pp. 61 y ss.

(25) PEREZ ROYO, J. Las fuentes de Derecho. Tecnos, Madrid, 1984.

(26) El principal impulsor de esta teoria fue Kelsen, aunque su positivismo jurídico no se siga con igual entusiasmo en doctrina. KELSEN, H. Teoria pura del Derecho. 
“Artículo 87. - La Constitución prevalece sobre toda otra norma legal. La ley sobre toda otra norma de inferior categoría, y así sucesivamente de acuerdo a su jerarquía jurídica".

Como hemos visto, los tratados internacionales, por regla general, poseen un valor normativo equivalente al de las leyes y, por ende, son normas inferiores a la Constitución. La cuestión, por lo tanto, consiste en determinar si puede ingresar válidamente un tratado en el ordenamiento interno, aún en el caso de contener disposiciones opuestas a la Constitución; en segundo término, admitiendo tal posibilidad, se debe indagar sobre lo que el propio texto constitucional establece para estos casos.

Orientándonos por la sistematización utilizada por De la Lama (27), las posturas en el derecho constitucional comparado se agrupan básicamente en alguna de las tres posibilidades o vías de solución siguientes:

Un primer tipo de regulación constitucional se caracteriza por otorgar una primacía relativa al tratado por encima de la Constitución. Aquí no existe un procedimiento diverso al utilizado normalmente para aprobar un tratado, pues el propio tex to constitucional es el que explícitamente establece esta prevalencia (28). Esta postura, muy poco utilizada, es la expresada por la Constitución del Reino de los Países Bajos:

"Artículo 63. - Si el desarrollo del orden legal internacional lo requiere, el contenido de un tratado puede apartarse de ciertas disposiciones de la Constitución" (29).

(27) DE LA LAMA E., M. La Constitución politica. . op. cit. p. 487 y ss.

(28) La norma de derecho internacional no es, per se superior al derecho interno, dado que su prevalencia se basa, precisamente, en la aplicación de las normas de derecho interno que regulan su valor normativo.

(29) Lsta previsión constitucional ha sido utilizada para admitir la trajafe rencia de competencias hacia la Comunidad Europea. 
Una segunda alternativa de solución se deriva de la simple aplicación del principio de jerarquía normativa. Así, el tratado que colisiona con la Constitución no puede ser aprobado. Esta es la opción más difundida y condiciona la aprobación de un tratado a su plena compatibilidad constitucional.

La última de las vías generalmente utilizadas en el derecho constitucional comparado permite que, previa modificación constitucional operada en la norma contradictoria, se apruebe el tratido. Del mismo modo que en el caso anterior, el tratado que colisiona con el texto constitucional no puede ingresar en el derecho in terno, pero, en cambio, establece la vía para remover el obstáculo que significa esta colisión normativa, es decir, la reforma constitucional.

La Constitución peruana tiene la virtud de incorporar una nueva modalidad de respuesta al problema suscitado por una colisión entre un tratado y la Constitución. Esta formulación, alejada de las antes reseñadas, está consignada de la forma siguiente:

"Artículo 103.- - Cuando un tratado internacional contiene una estipulación que afecta una disposición constitucional, debe ser aprobado por el mismo procedimiento que rige la reforma de la Constitución, antes de ser ratificado por el Presidente de la República”.

En estos casos, el procedimiento que la Constitución establece para la aprobación de los tratados es sustituido por el de la reforma constitucional. En realidad, el Parlamento se manticne como el órga no competente para aprobar estos tratados, pero deberá seguir un procedimiento más dilatado y riguroso. En sintesis. de acuerdo al artículo 306 de la Carta, el tratado "debe se' aprobado en una Primera Legislatura Ordinaria 1 ratificado en otra Primera Legislatura Ordinaria consecutiva"; por otro lado: " aprobación y la ratificación - se refiere a la segunda aprobaciónrequieren la mavoria absoluta de los votos del número legal de miembros de cada una de las Cámaras".

Debemos notar que no se trata de una simple variación de la 
tercera de las vías de solución mencionadas. En efecto, mientras que en un caso el texto constitucional es modificado y, por ende, sólo una nueva reforma puede poner en vigencia la norma modificada, en la fórmula peruana no existe ninguna reforma constitucional. Asi, en el primer caso existe una modificación o derogación de la norma constitucional y, en el caso peruano, lo que opera es una restricción en los campos de aplicación de la norma, "ello implica que la norma constitucional seguiría rigiendo fuera del ámbito de aplicación del tratado y recuperaría su plena vigencia en caso que éste llegara a terminar" (30). Como consecuencia, la sustitución de la norma constitucional pór la del tratado, sólo se verifica sobre el campo de aplicación del tratado. En todos los demás casos la Constitución mantiene plena vigencia y aplicabilidad.

El modelo que consagra la Constitución peruana presenta algunas ventajas respecto de los que enumeramos inicialmente. Así por ejemplo, en el caso de la denuncia o de la expiración del plazo de un tratado, la plena entrada en vigor de la norma constitucional es automática. Por el contrario, en este mismo supuesto, las vias que exigen la reforma constitucional previa deberán realizar una nueva reforma para lograr la vigencia de la norma modificada, de modo que a pesar de la desaparición de la causa de la reforma, vale decir, el tratado opuesto a la Constitución, tales efectos perdurarán hasta que se haya completado el dilatado y agravado proceso de reforma constitucional.

Por otro lado, las vías que establecen la previa y necesaria reforma constitucional presentan un problema adicional. En efecto, puede ocurrir que la norma constitucional sea efectivamente reformada y que, sin embargo, el tratado que origina la reforma no entre aún en vigencia y ésta no dependa de la voluntad de tal Estado; esto puede darse, por ejemplo, cuando se exige como requisito el depósito de un determinado número de instrumentos; en el extremo de los casos, puede que la vigencia se vea postergada por un largo período o que el tratado nunca llegue a entrar en

(30) DE LA LAMA E., M. La Constitución politica. . op. cit. p. 487. 
vigor, mientras que la reforma constitucional surte plenos efectos jurídicos, creándose un desfase normativo.

\section{La supremacia del Derecho Internacional en la Constitución de 1979}

Apoyándose en las diversas normas que regulan los tratados de nuestro texto constitucional, el profesor De la Lama ha concluido en que la Carta Fundamental peruana "presenta una clara tendencia monista, reconociendo la primacia del derecho internacional convencional sobre el derecho interno peruano" (31). La primacía del derecho internacional sobre el derecho interno ha sido defendida por los tribunales internacionales y constituye la piedra angular de su eficacia. El profesor Miaja de la Muela (32) realiza una exhaustiva investigación de los argumentos que sostienen y evidencian la necesidad de esta supremacía, entre los que extraemos uno particularmente clarificador: "no se concibe un Derecho Internacional merecedor de este nombre, si no se afirma superior al Derecho dictado por los Estados, destinatarios de las normas de aquél".

Al respecto es pertinente efectuar una distinción entre el derecho internacional general y el convencional, pues es en este último donde se ha experimentado los mayores avances. En efecto, la Convención de Viena de 1969, particularmente el artículo 27, nos sitúa, en palabras de Sánchez Rodríguez (33), "en presencia del principio general de prevalencia de las normas contenidas en los tratados sobre las normas propias del ordenamiento interno de los Estados obligados por aquéllos".

(31) DE LA LAMA E., M. La Constitución politica. . op. cit. p. 491

(32) MIAJA de la Muela, A. La primacia sobre los ordenamientos juridicos internos del Derecho Internacional y del Derecho Comunitario Europeo. En: Revista de Instituciones Europeas, Vol. 1 a 3, agosto-diciembre de 1974, p. 1001 y ss.

(33) SANCHEZ RODRIGUEZ, L.I. El proceso de celebración de los tratados internacionales y su eficacia interna en el sistema constitucional español (Teoria y práctica). Madrid, 1974, p. 23. 
No debemos olvidar, sin embargo, que el citado artículo 27 esta, a su vez. recogido en un tratado, lo que significa que se trata de una norma de derecho internacional convencional, por lo que, eventualmente, podría seguir el mismo destino que las normas que pretende regular. Ello no hace sino dotar de mayor importancia el afán del constituyente peruano, al establecer expresamente la supremacía de las normas internacionales en el texto constitucional. En tal sentido, la obligada observancia de esta prevalencia por parte de los órganos del Estado, especialmente de los jueces, no fluye de un tratado o de un principio de derecho internacional, sino de la norma de mayor jerarquía en el derecho interno. También se ha destacado que "hay que distinguir la incorporacion del tratado al derecho interno con rango de lev o con jerarquil superior a la ley. La diferchcia es clara, puesto que en el primer caso el tratado podría ser derogado o modificado por una ley posterior, hipótesis imposible si se reconociese la supremacia del tratado sobre la le.." (34).

Sobre ello, como hemos podido comprobar, la Constitución peruana ha logrado el efecto deseado, sin que la fórmula sea estrictamente jerárquica. El tratado posee jerarquía igual a la ley, elevándose por encima de las normas subordinadas, pero además tiene prevalencia sobre las normas con rango de ley que le sean opuestas. La ley no deroga ni modifica al tratado. El tratado logra la inaplicación de la ley en todo lo que le es opuesta y en el campo de su aplicación, pero no la deroga, pues mantiene vigencia en los demás supuestos ajenos al tratado. Sin dejar de advertir la importancia de estas disposiciones respecto de las obligaciones ad extra del Estado, su principal virtud se aprecia en las posibilidades que son extendidas al particular para invocar y exigir, en su caso, la observancia y aplicación preferente de las normas convencionales de derecho in ternacional.

La prevalencia otorgada al derecho convencional internacional no se reduce al reconocimiento de su jerarquía de ley, con apli-

(34) MIAJA DE LA MUELA, A. La primacia sobre los ordenamientos... op. cit. p. 1000. 
cación preferente. La carta constitucional peruana permite que tratados que le sean opuestos puedan ingresar en la esfera del derecho interno, disponiendo como único requisito la utilización del procedimiento de reforma para su aprobación en las cámaras. Incluso, respecto de las normas de tratados relativas a los derechos humanos, la regulación constitucional otorga jerarquía constitucional sin que sea preciso observar el requisito de procedimiento anterior. Este conjunto de normas constitucionales, más allá de los problemas que puedan generar dentro del sistema de fuentes de derecho interno, configuran el marco y el fundamento jurídico, necesarios para preservar la vigencia del derecho convencional internacional, sobre la base de reconocer su prevalencia sobre las normas internas. 